\title{
APPROXIMATION THEOREMS FOR UNIFORMLY CONTINUOUS FUNCTIONS
}

\author{
BY \\ ANTHONY W. HAGER(')
}

\begin{abstract}
Let $X$ be a set, $A$ a family of real-valued functions on $X$ which contains the constants, $\mu_{A}$ the weak uniformity generated by $A$, and $U\left(\mu_{A} X\right)$ the collection of uniformly continuous functions to the real line $R$. The problem is how to construct $U\left(\mu_{A} X\right)$ from $A$. The main result here is: For $A$ a vector lattice, the collection of suprema of countable, finitely $A$. equiuniform, order-one subsets of $A^{+}$is uniformly dense in $U\left(\mu_{A} X\right)$. Two less technical corollaries: If $A$ is a vector lattice (resp., vector space), then the collection of functions which are finitely A-uniform and uniformbly locally-A (resp., uniformly locally piecewise-A) is uniformly dense in $U\left(\mu_{A} X\right)$. Further, for any $A, a$ finitely $A$-uniform function is just a compasition $F \circ\left(a_{1}, \ldots, a_{p}\right)$ for some $a_{1}, \ldots, a_{p} \in A$ and $F$ uniformly continuous on the range of $\left(a_{1}, \ldots, a_{p}\right)$ in $R^{p}$. Thus, such compositions are dense in $U\left(\mu_{A} X\right)$. For $B U\left(\mu_{A} X\right)$, the compositions with $F \in B U\left(R^{p}\right)$ are dense (B denoting bounded functions). So, in a sense, to know $U\left(\mu_{A} X\right)$ it suffices to know $A$ and subspaces of the spaces $R^{p}$, and to know $B U\left(\mu_{A} X\right), A$ and the spaces $R^{p}$ suffice.
\end{abstract}

In case $A$ is a vector lattice and $A=B A$ (i.e., $A$ consists of bounded functions), the problem of describing $U\left(\mu_{A} X\right)$ in terms of $A$ has an elegant solution: $A$ is uniformly dense in $U\left(\mu_{A} X\right)$ (i.e., if $f \in U\left(\mu_{A} X\right)$ and $\varepsilon>0$ there is $a \in A$ with $|f(x)-a(x)|<\varepsilon$ for all $x \in X)$. This can be seen to be essentially equivalent to the usual Stone-Weierstrass Theorem. The result appears to have been first published in 1955 by Maak [M] and Nöbeling and Bauer [NB]; we give a short proof in the course of proving our main theorem.

If $A$ contains unbounded functions, the situation is more complicated: $A=U(R) \mid N$ is a "uniformly closed" vector lattice, while $\mu_{A}$ is (uniformly) discrete. With $X=R$, let $A$ be the piecewise linear functions (finitely many pieces); $\mu_{A}$ is the usual uniformity on $R$ and the closure of $A$ consists only of continuous eventually linear functions.

Received by the editors October 28, 1975 and, in revised form, August 10, 1976.

AMS (MOS) subject classifications (1970). Primary 41A30, 46E05, 54C30, 54E05; Secondary 06A65, 46A40, 54B99.

Key words and phrases. Stone-Weierstrass, uniformly continuous, uniform approximation.

(') The author is pleased to thank the Academies of Sciences of Czechoslovakia and the United States for support, J. R. Isbell and the referee for suggesting the formulation in terms of piecewise- $A$ functions in $\$ 3$ below, and W. L. Reddy for some helpful advice on the paper.

Preliminary versions of the main result were presented at the Pittsburgh Symposium, December, 1972 (see [H]) and to the Prague seminar on uniform spaces, May, 1975. 
The theorem that we prove shortly derives from the usual StoneWeierstrass Theorem (or the one mentioned above) and some results of Fenstad [F] and Császár [C] giving conditions sufficient for density and characterizations of the structures $U(\mu X)$. See 1.5 below.

1. The main theorem. This is 1.3 below, which explicitly, if rather technically, constructs $U\left(\mu_{A} X\right)$ from $A$. We require some preliminaries.

We make the standing assumption that $A$ is a subset of $R^{X}$ which contains the constants. Saying that $A$ is a vector space or vector lattice refers to the pointwise operations.

$\mu_{A}$ denotes the weak uniformity generated by $A$, that is, the smallest uniformity such that each function in $A$ is uniformly continuous. If $\delta(\varepsilon)$ denotes the cover of $R$ consisting of $\varepsilon$-balls, then $\{\delta(\varepsilon) \mid \varepsilon>0\}$ is a base for the usual uniformity of $R$ (using the covering description of uniformities [I(2)]). Thus, a subbase for $\mu_{A}$ is $\left\{f^{-1} \delta(\varepsilon) \mid f \in A, \varepsilon>0\right\}$.

Thus, a family $\mathscr{F} \subset R^{X}$ is $A$-equiuniform (i.e., equiuniform for $\mu_{A}$ ) if given $\varepsilon>0$, there is $\mu_{A}$-basic cover $\mathscr{B}$ such that whenever $B \in \mathscr{B}$ and $x, y \in B$, then $|f(x)-f(y)|<\varepsilon$ for all $f \in \mathscr{F}$. This means that given $\varepsilon>0$ there are $\left\{a_{1}, \ldots, a_{p}\right\} \subset A$ and $\delta>0$ such that whenever $\left|a_{i}(x)-a_{i}(y)\right|<\delta$ for $i=1, \ldots, p$, then $|f(x)-f(y)|<\varepsilon$ for all $f \in \mathscr{F}$-since basic $\mathscr{B}$ is of the form $\wedge_{i=1}^{p} a_{i}^{-1} \delta(\delta)$.

In this definition, if the family $\left\{a_{1}, \ldots, a_{p}\right\}$ may be chosen independently of $\varepsilon$, we shall call $\mathscr{F}$ finitely-A-equiuniform. Evidently, this means that there is finite $F \subset A$ such that $\mathscr{F}$ is $F$-equiuniform.

As is well known, if $\mathscr{F} \subset R^{X}$ is $\mu$-equiuniform (for a uniformity $\mu$ on $X$ ), and if the pointwise supremum $\bigvee \mathscr{F}$ is finite at each point, then $\bigvee \mathscr{F} \in$ $U(\mu X)$. We shall need a generalization:

1.1 LEMMA. If $\mathscr{F}$ is finitely-A-equiuniform, using finite $F \subset A$, with $\bigvee \mathscr{F}$ finite at each point, and if $\mathcal{S}$ is any collection of subsets of $\mathscr{F}$, then $\{\bigvee S \mid S \in$ $\delta$ \} is finitely-A-equiuniform, and using $F$.

This is an immediate consequence of the inequality [F(1)]: for $\mathscr{B}, \mathcal{C} \subset R$, $|\bigvee \mathfrak{B}-\bigvee \mathfrak{C}|<\bigvee\{|b-c| \mid b \in \mathscr{B}, c \in \mathcal{C}\}$

Finally, a family of sets will be said to be of order one if each three members have empty intersection. We shall deal with families $\mathscr{F} \subset R^{x}$ for which $\operatorname{coz} \mathscr{F}$ is a cover of order one; here, $\operatorname{coz} \mathscr{F}=\{\operatorname{coz} f \mid f \in \mathscr{F}\}$ and $\operatorname{coz} f=\{x \in X \mid f(x) \neq 0\}$.

1.2 Notation. $A_{0}$ stands for the collection of functions of the form $\bigvee \bar{F}$, where

(a) $\mathscr{F} \subset A^{+}$,

(b) $\mathscr{F}$ is countable,

(c) $\mathscr{F}$ is finitely- $A$-equiuniform, 
(d) $\operatorname{coz} \mathscr{F}$ is a $\mu_{A}$-uniform cover of order one.

1.3 THEOREM. If $A$ is a vector lattice, then $A_{0}$ is uniformly dense in $U\left(\mu_{A} X\right)^{+}$.

The proof of 1.3 is a bit involved. We first sketch it for an almost prototypical special case, and give a corollary.

1.4 Let $X=[0,+\infty)$, with $A$ the vector lattice of continuous piecewise linear functions (with finitely many pieces). Here, $\mu_{A}$ is the usual uniformity.

Given $f \in U\left(\mu_{A} X\right)^{+}$, and $\varepsilon>0$, choose $\delta>0$ and uniformly continuous $g$ which is linear on each interval $[n \delta,(n+1) \delta] \equiv I_{n}$ and which approximates $f$ within $\varepsilon$. By uniform continuity, a number $s>0$ can be chosen so that $g_{n}<g$ for each $n$, where $g_{n}$ is defined like this: let $L_{n}(x)$ be linear of slope $s$ with $L_{n}(n \delta)=g(n \delta), M_{n}(x)$ linear of slope $-s$ with $M_{n}((n+1) \delta)=$ $g((n+1) \delta)$; then $L_{n}\left|I_{n}=g\right| I_{n} ;$ for $x<n \delta, g_{n}=0 \vee L_{n}$; for $x>(n+1) \delta$, $g_{n}=M_{n} \vee 0$.

Clearly, $\bigvee_{n} g_{n}=g$, and $\left\{g_{n}\right\}$ satisfies 1.2(a), (b), and (c) using $\left\{a_{1}, \ldots, a_{p}\right\}=$ the singleton $\left\{a_{1}(x)=s x\right\}$. But (d) does not yet hold. To achieve (d), define inductively $K_{1}<K_{2}<\ldots$ in such a way that (d) holds for

$$
f_{1}=\bigvee\left\{g_{n} \mid K_{i}+1 \leqslant n<K_{i+1}\right\} .
$$

This is done by making the differences $\left|K_{i+1}-K_{i}\right|$ grow rapidly. Then $\bigvee_{i} f_{i}=\bigvee_{n} g_{n}$, and $\left\{f_{i}\right\}$ is finitely $A$-equiuniform by 1.1 .

1.5 COROLLARY. Let $A$ be a vector lattice.

(a) If $A_{0}=A^{+}$, then $A$ is uniformly dense in $U\left(\mu_{A} X\right)$.

(b) $A=U\left(\mu_{A} X\right)$ iff $A_{0}=A^{+}$and $A$ is "uniformly closed".

Proof. (a) Given $f \in U\left(\mu_{A} X\right)$, write $f=f^{+}-f^{-}$. By 1.3, approximate $f^{+}$ and $f^{-}$by $g$ and $h$ in $A_{0}=A^{+}$. Then $g-h \in A$, and approximates $f$.

(b) Any $U(\mu X)$ is closed under taking uniform limits and taking sups of families $\mathscr{F}$ satisfying 1.2(c). The converse follows from (a).

(a) can be viewed as an improvement of a combination of theorems of Fenstad and Császár. [F] $A$ is dense in $U\left(\mu_{A} X\right)$ if $A$ is closed under the taking of suprema of countable, equiuniform, star-finite families. (1.2(d) implies $\mathscr{F}$ is star-two. The result here is a combination of 4.1 of $[\mathrm{F}(1)]$ and 4.3 of [F(2)]; see also [H].) [C] $A$ is dense in $U\left(\mu_{A} X\right)$ if $A$ is closed under the taking of pointwise limits of finitely equiuniform sequences. (This is approximately Satz 3 of $[\mathbf{C}]$.) Here note that: If $\left\{f_{n}\right\}$ is a finitely equiuniform sequence, then $g_{n} \equiv \bigvee_{k<n} f_{k}$ defines a sequence $\left\{g_{n}\right\}$ which converges pointwise to $\bigvee_{n} f_{n}$, and which is finitely equiuniform by 1.1; thus Császár's condition implies that $A_{0}=A^{+}$. 
Fenstad and Császár also derive corollaries like (b), which similarly (b) improves.

Proof of 1.3. If $\mathscr{F}$ satisfies 1.2 , then $\mathscr{F}$ is equiuniform for $\mu_{A}$, and $\bigvee \mathscr{F} \in U\left(\mu_{A} X\right)$. So $A_{0} \subset U\left(\mu_{A} X\right)$.

For the density: Let $\delta(c, r)$ be the open interval in $R$ with center $c$ and radius $r$. Let $\mathcal{L}(r)=\{S(n r, r) \mid n \in Z\}$. Evidently, $\{\mathcal{L}(r) \mid r>0\}$ is a base for the (usual) uniformity of $R$, and $\left\{a^{-1} \mathcal{L}(r) \mid a \in A, r>0\right\}$ is a subbase for $\mu_{A}$.

Given $f \in U\left(\mu_{A} X\right)^{+}$and $\varepsilon>0$, choose a basic cover $\mathcal{Q}=\bigwedge_{i=1}^{P} a_{i}^{-1} \mathcal{L}(\delta)$ $<f^{-1} \mathcal{L}(\varepsilon)$. Members of $\mathcal{Q}$ will be denoted $\alpha, \beta, \gamma, \ldots$

Given $\alpha=\cap_{i=1}^{p} a_{i}^{-1} S\left(c_{i}, \delta\right)$,

(1) define $n(\alpha) \equiv$ least integer $n$ with $\alpha \subset f^{-1} S(n \varepsilon, \varepsilon)$, and

$$
e(\alpha)(x) \equiv 2-2 \wedge \frac{1}{\delta}\left[Y\left|a_{i}(x)-c_{i}\right|\right] \vee 1
$$

Then

$$
|\bigvee \varepsilon n(\alpha) e(\alpha)(x)-f(x)|<2 \varepsilon \text { for all } x \in X
$$

This will follow from

$$
\begin{aligned}
e(\alpha)(x) & =1 \quad \text { iff }\left|a_{i}(x)-c_{i}\right|<\delta / 2 \text { for each } i ; \\
e(\alpha)(x) & =0 \text { iff }\left|a_{i}(x)-c_{i}\right|>\delta \text { for some } i \\
0 & <e(\alpha)<1, \quad \text { and } \operatorname{coz} e(\alpha)=\alpha .
\end{aligned}
$$

(3) If $e(\alpha)(x) \neq 0$, then $e(\beta)(x)=1$ for some $\beta$ with $\beta \cap \alpha \neq \varnothing$.

(4) If $\beta \cap \alpha \neq \varnothing$ then $n(\beta)<n(\alpha)+1$.

Proofs. (2) is computed straightforwardly. (4) follows from the facts that $\mathbb{Q}<f^{-1} \mathcal{L}(\varepsilon)$ and the latter is star-two.

For (3), if $e(\alpha)(x)=1$ then $\beta=\alpha$ works, so suppose that $0<e(\alpha)(x)<1$. Then both inequalities in (2) are violated: $\left|a_{j}(x)-c_{j}\right|>\delta / 2$ for some $j$, and $\left|a_{i}(x)-c_{i}\right|<\delta$ for each $i$. Then let

$$
\begin{aligned}
& K_{j}^{\prime}=K_{j} \pm \delta, \text { in such a way that }\left|a_{j}(x)-c_{j}\right|<\delta / 2, \\
& K_{i}^{\prime}=K_{i} \text { or } K_{i} \pm \delta, \text { so that }\left|a_{i}(x)-c_{i}\right|<\delta / 2 .
\end{aligned}
$$

Set $\beta=\cap_{i} a_{i}^{-1} S\left(K_{i}^{\prime}, \delta\right)$; then $\beta \cap \alpha \neq \varnothing$ and $e(\beta)(x)=1$.

Proof of (1). Given $x, x \in f^{-1} S(n \varepsilon, \varepsilon)$ for at most two consecutive $n$ 's. Let $n$ be the first, so that $n \varepsilon-\varepsilon<f(x)<(n+1) \varepsilon+\varepsilon$.

If $e(\alpha)(x) \neq 0$ then $x \in \alpha$ and $n<n(\alpha)<n+1$. So first: $V_{\alpha} \varepsilon n(\alpha) e(\alpha)(x)<\varepsilon(n+1) \cdot 1$. And second: there is $\alpha$ with $x \in \alpha$ so (by (4)) there is $\beta$ with $e(\beta)(x)=1$. Since $x \in \beta, n<n(\beta)$, and

$$
\varepsilon n<\varepsilon n(\beta) e(\beta)(x)<\bigvee_{\alpha} \varepsilon n(\alpha) e(\alpha)(x) .
$$

Digression. (a) If $A=B A$, then $A$ is dense in $U\left(\mu_{A} X\right)$. We have 
essentially shown this: By writing arbitrary $f \in U\left(\mu_{A} X\right)$ as $f=f^{+}-f^{-}$, we see that it suffices to approximate functions in $U\left(\mu_{A} X\right)^{+}$. Given such $f$ and $\varepsilon$, proceed as above. Because each $a_{i}$ is bounded, $\mathbb{Q}=\{\alpha\}$ is finite. Thus $\bigvee_{\alpha} \varepsilon n(\alpha) e(\alpha) \in A$ and (1) applies.

(b) In the general case, the approximation in (1) is only preliminary. Clearly, $\{e(\alpha) \mid \alpha \in \mathbb{Q}\}$ is finitely equiuniform (using $\left\{a_{1}, \ldots, a_{p}\right\}$ ), but not so for $\{\varepsilon n(\alpha) e(\alpha) \mid \alpha \in \mathbb{Q}\}$ which, roughly speaking, grow from 0 to $\varepsilon n(\alpha)$ nonuniformly in $\alpha$; also, $e(\beta) \wedge e(\alpha) \neq 0$ for possibly $3^{p}-1$ other $\beta^{\prime}$ 's because $\operatorname{coz} e(\alpha)=\alpha$ (from (2)), and $\mathcal{Q}$ has the same "starring" properties as the cover $\bigwedge_{i=1}^{p} \pi_{i}^{-1} \mathcal{L}(\alpha)$ of $R^{p}$.

So we shall, first, spread out the support of each $\varepsilon n(\alpha) e(\alpha)$ so as to achieve equiuniformity, and then do some collecting together as in 1.4 to make the supports of order one.

Given $\alpha$, let $\alpha_{0}=\alpha, \alpha_{1}=\cup\{\beta \in \mathbb{Q} \mid \beta \cap \alpha \neq \varnothing\}$, and $\ldots \alpha_{i}=\cup\{\beta$ $\left.\in \mathbb{Q} \mid \beta \cap \alpha_{i-1} \neq \varnothing\right\}$. As noted earlier, the "starring" properties of $\mathcal{Q}$ are those of $\wedge_{i=1}^{p} \pi_{i}^{-1} \mathcal{L}(\delta)$ in $R^{p}$ : so each $\alpha$ meets $<3^{p}$ members of $Q$; note that $3^{p}$ is the volume of a cube in $R^{p}$ of side $3 .\left|\left\{\beta \mid \beta \cap \alpha_{1} \neq \varnothing\right\}\right|<$ the volume of the cube in $R^{p}$ of side 5 , and, in general, $\left|\left\{\beta \mid \beta \cap \alpha_{i-1} \neq \varnothing\right\}\right|<(i+2)^{p}$, which is the volume of the cube of side $(i+2)$.

Let $e\left(\alpha_{i}\right) \equiv \bigvee\left\{e(\beta) \mid \beta \subset \alpha_{i}\right\}$. There are $<(i+2)^{p}$ such $\beta$ 's, so $e\left(\alpha_{i}\right) \in$ $A$. Moreover, $\left\{e\left(\alpha_{i}\right) \mid \alpha \in \mathbb{Q}, i=0,1,2, \ldots\right\}$ is finitely $A$-equiuniform by 1.1.

(5) Let $f(\alpha) \equiv 2 \varepsilon \Sigma\left\{e\left(\alpha_{i}\right) \mid 0<i<n(\alpha) / 2\right\}$; then $\{f(\alpha) \mid \alpha \in \mathbb{Q}\}$ is finitely $A$-equiuniform, and $\left|\bigvee_{a} f(\alpha)(x)-f(x)\right|<3 \varepsilon$ for each $x \in X$.

Note that $f(\alpha)$ is approximately $\varepsilon n(\alpha) e(\alpha)$ on the set $\alpha$, but the jump in $e n(\alpha) e(\alpha)$ has been spread over

$$
\operatorname{coz} f(\alpha)=\cup\left\{\operatorname{coz} e\left(\alpha_{i}\right) \mid 0<i<\frac{n(\alpha)}{2}\right\}=U\left\{\alpha_{i} \mid 0<i<\frac{n(\alpha)}{2}\right\} .
$$

To prove (5), observe that

$$
e\left(\alpha_{i+1}\right) \mid \alpha_{i} \equiv 1 ; \quad \beta \subset \alpha_{i} \text { implies } n(\beta)<n(\alpha)+i .
$$

Proof. The first is implied by (3), and the second follows by iterating (4).

Proof OF (5). Given $\varepsilon_{0}>0$, choose $\delta>0$ so that whenever $\left|a_{i}(x)-a_{i}(y)\right|$ $<\delta$ for $i=1, \ldots, p$, then (i) whenever $x \in \alpha$ then $y \in \alpha_{1}$, and if $y \in \alpha$ then $x \in \alpha_{1}$, and (ii) $\left|e\left(\alpha_{i}\right)(x)-e\left(\alpha_{i}\right)(y)\right|<\varepsilon_{0}$ for every $\alpha_{i}$. Suppose $x$ and $y$ are "this close".

Given $\alpha$ : if $f(\alpha)(x)=f(\alpha)(y)=0$, there is nothing to prove. Suppose, say, that $f(\alpha)(x) \neq 0$, and let $i$ be the least integer $<n(\alpha) / 2$ with $e\left(\alpha_{i}\right)(x) \neq 0$. Then $e\left(\alpha_{i+1}\right)(x)=1$. We then have: If $j<i, e\left(\alpha_{j}\right)(x)=0$; so if $j<i-1$, $e\left(\alpha_{j}\right)(y)=0$. If $j>i, e\left(\alpha_{j}\right)(x)=1$; so if $j>i+1, e\left(\alpha_{j}\right)(y)=1$. Thus, 


$$
\begin{aligned}
|f(\alpha)(x)-f(\alpha)(y)| & =2 \varepsilon\left|\sum_{j}\left\{e\left(\alpha_{j}\right)(x)-e\left(\alpha_{j}\right)(y)\right\}\right| \\
& =2 \varepsilon\left|\sum\left\{e\left(\alpha_{j}\right)(x)-e\left(\alpha_{j}\right)(y) \mid j=i-1, i, i+1\right\}\right| \\
& <2 \varepsilon \cdot 3 \cdot \varepsilon_{0} .
\end{aligned}
$$

For present purposes, $\varepsilon$ is fixed; so $\{f(\alpha) \mid \alpha \in A\}$ is finitely $A$-equiuniform.

Next, we shall show that $\left|\bigvee_{\alpha} f(\alpha)(x)-\bigvee_{\alpha} \varepsilon n(\alpha) e(\alpha)(x)\right|<\varepsilon$ for all $x \in$ $X$. Then (1) will apply.

We always have $e(\alpha)<e\left(\alpha_{i}\right)$, and the number of terms in $f(\alpha)$ is at least $(n(\alpha)-1) / 2$. So

$$
\varepsilon(n(\alpha)-1) e(\alpha)<2 \varepsilon\left(\frac{n(\alpha)-1}{2}\right) e(\alpha)<2 \varepsilon \sum e\left(\alpha_{i}\right)=f(\alpha) .
$$

Thus for any $\alpha, \varepsilon n(\alpha) e(\alpha) \leqslant f(\alpha)+\varepsilon$, and thus $\bigvee_{\alpha} \varepsilon n(\alpha) e(\alpha) \leqslant \bigvee_{\alpha} f(\alpha)+$ $\varepsilon$.

Now, given $\alpha$ and $x \in X$, we show there is $\beta$ with $f(\alpha)(x)<\varepsilon n(\beta) e(\beta)+$ e. Then $\bigvee_{a} f(\alpha)<\bigvee_{\alpha} \varepsilon n(\alpha) e(\alpha)+\varepsilon$ follows. If $f(\alpha)(x)=0$, then take $\beta=$ $\alpha$. Otherwise, there is least $i<n(\alpha) / 2$ with $e\left(\alpha_{i}\right)(x) \neq 0$, and

$$
\begin{aligned}
f(\alpha)(x) & =2 \varepsilon \sum\left\{e\left(\alpha_{j}\right)(x) \mid i<j \leqslant n(\alpha) / 2\right\} \\
& <2 \varepsilon(n(\alpha) / 2-i)=\varepsilon(n(\alpha)-2 i) .
\end{aligned}
$$

Choose $\beta \subset \alpha_{i+1}$ with $e(\beta)(x)=1$, so $\alpha \subset \beta_{i+1}$ as well, and $n(\alpha) \leqslant n(\beta)+$ $(i+1)$. Then

$$
n(\alpha)-2 i \leqslant n(\alpha)-i \leqslant n(\beta)+1 \text {. }
$$

Thus $f(\alpha)(x)<\varepsilon(n(\beta)+1)=\varepsilon n(\beta) e(\beta)(x)+\varepsilon$.

We now complete the construction by making the supports of order one.

$Q$ breaks into equivalence classes of the relation $\beta \sim \alpha$ if $\beta \subset \alpha_{i}$ for some positive integer $i$. Let $R$ consist of one representative from each class.

Given $\alpha \in \Re$ and $\beta \sim \alpha$, let $d(\beta)=$ the least $i$ with $\beta \subset \alpha_{i}$. We shall determine a sequence $K_{1}<K_{2}<\ldots$ of positive integers (perhaps finite, and depending on $\alpha$ ) so that with

$$
f_{i}(\alpha) \equiv \bigvee\left\{f(\beta) \mid K_{i}+1<d(\beta)<K_{i+1}\right\},
$$

the family $\left\{\operatorname{coz} f_{i}(\alpha) \mid i=1,2, \ldots\right\}$ is of order one. Note that according to the remarks preceding (5),

$$
\left|\left\{\beta \mid \beta \subset \alpha_{K_{i+1}}\right\}\right|<\left(K_{i+1}+2\right)^{p} \text {, so that each } f_{i}(\alpha) \in A .
$$

To define $\left\{K_{i}\right\}$ precisely, we use

(8) If $f(\beta) \wedge f(\alpha) \neq 0$, then $\beta \subset \alpha_{2 n(\alpha)+3}$.

PROOF. If $f(\beta) \wedge f(\alpha) \neq 0$, then there are $\gamma$ and $i \leqslant n(\alpha) / 2, j<n(\beta) / 2$ 
with $\gamma \subset \alpha_{i+1} \cap \beta_{j+1}$. Then $\beta \subset \gamma_{j+1} \subset\left(\alpha_{i+1}\right)_{j+1}=\alpha_{i+j+2}$, and thus

$$
n(\beta)<n(\alpha)+i+j+2 \leqslant n(\alpha)+n(\alpha) / 2+n(\beta) / 2+2
$$

(using (6)). Solving for $n(\beta), n(\beta)<3 n(\alpha)+1$. Thus

$$
i+j+2<n(\alpha) / 2+\left(3 n(\alpha) / 2+\frac{1}{2}\right)+2<2 n(\alpha)+3,
$$

and $\beta \subset \alpha_{2 n(\alpha)+3}$

Now, what is required of $\left\{K_{i}\right\}$ is that $f(\beta) \wedge f(\gamma)=0$ whenever $d(\beta)>$ $K_{i+1}$ and $d(\gamma)<K_{i} \cdot d(\gamma) \leqslant K_{i}$ implies $n(\gamma)<n(\alpha)+K_{i}$ (by (6)), so if $f(\beta) \wedge f(\gamma) \neq 0$ then (using (8)),

$$
\beta \subset \gamma_{2 n(\gamma)+3} \subset\left(\alpha_{K_{i}}\right)_{2\left(n(\alpha)+K_{i}\right)+3}=\alpha_{2 n(\alpha)+2 K_{i}+3}
$$

So take $K_{1}=1$, and inductively, $K_{i+1}=2 n(\alpha)+2 K_{i}+3$. Then $\left\{\operatorname{coz} f_{i}(\alpha) \mid i\right.$ $=1,2, \ldots\}$ is of order one.

Let $\mathscr{F} \equiv\left\{f_{i}(\alpha) \mid \alpha \in \Re ; i=1,2, \ldots\right\}$. If $\alpha \neq \beta$ in $\mathscr{R}$, then $f_{i}(\alpha) \wedge f_{j}(\beta)$ $=0$; so $\operatorname{coz} \mathscr{F}$ is also of order one. To see that it is a $\mu_{A}$-uniform cover: Fix $\alpha$. Then

$$
\left\{\operatorname{coz} f_{i}(\alpha)\right\}_{i}>\{\operatorname{coz} f(\beta) \mid \beta \sim \alpha\}>\{\operatorname{coz} e(\beta) \mid \beta \sim \alpha\}>\{\beta \mid \beta \sim \alpha\} .
$$

Thus $\operatorname{coz} \mathcal{F}$ is refined by the $\mu_{A}$-uniform cover $\mathcal{Q}$ consisting of all $\alpha$ 's.

By (5) and 1.1, $\mathcal{F}$ is finitely $A$-equiuniform. For each $\beta \in \mathbb{Q}, f(\beta)$ is a term in some $f_{i}(\alpha)$, so that $\bigvee \mathscr{F}=\bigvee\{f(\beta) \mid \beta \in \mathbb{Q}\}$; by (5) $\bigvee \mathcal{F}$ approximates $f$

2. Locally- $A$ functions. We present a less technical, perhaps more memorable, corollary of 1.3 , isolating a class of functions dense in $U\left(\mu_{A} X\right)$ with a considerably simpler description.

2.1 Definition. $A_{1}$ stands for the collection of functions $g \in R^{X}$ which are

(a) finitely $A$-uniform: $g \in U\left(\mu_{F} X\right)$ for some finite $F \subset A$; and

(b) uniformly locally- $A$ : there is a $\mu_{A}$-uniform cover on each member of which $g$ agrees with some function in $A$.

There are other ways to put (b). For example, each of the following is equivalent to " $g$ is uniformly locally- $A$ ": ( $\left.\mathrm{b}^{\prime}\right)$ There are $a_{1}, \ldots, a_{p} \in A$ and $\delta>0$ such that if $S$ is a subset of $X$ for which each osc $a_{i}<\delta$, then there is $a_{S} \in A$ with $g\left|S=a_{S}\right| S$. ( $\left.b^{\prime \prime}\right)$ There is a sequence $\left\{a_{n}\right\} \subset A$ such that $\left\{x \in X \mid g(x)=a_{n}(x)\right\}_{n}$ is a $\mu_{A}$-uniform cover.

$\$ 4$ will say more about the finitely $A$-uniform functions.

\subsection{THEOREM. If $A$ is a vector lattice, then $A_{1}$ is uniformly dense in $U\left(\mu_{A} X\right)$.}

Proof. First, $A_{0} \subset A_{1}$ : Let $\mathcal{F}$ be as in 1.2. By 1.1, $\bigvee \mathcal{F}$ is finitely $A$-uniform. By 1.2(d), $\operatorname{coz} \mathcal{F}$ is $\mu_{A}$-uniform, and on the member $\operatorname{coz} \mathrm{g}$, $\bigvee \mathscr{F}=\bigvee\{h \in \mathscr{F} \mid \operatorname{coz} h \cap \operatorname{coz} g \neq \varnothing\}$; the latter function is in $A$ because of the "order one" condition.

By 1.3, $A_{0}-A_{0}$ is dense in $U\left(\mu_{A} X\right)$; of course, $A_{0}-A_{0} \subset A_{1}-A_{1}$. But 
$A_{1}-A_{1}=A_{1}$, as is readily checked. (In fact, $A_{1}$ is a vector lattice, because $A$ is.)

2.3 REMARK. If in 2.2 (or 1.3) we assume that $A$ is only a vector space, then the conclusion fails: Consider the vector space $A$ of linear functions on $R$, for which $\mu_{A}$ is the usual uniformity. Any uniformly piecewise- $A$ function is locally linear, and an easy chaining argument shows that a locally linear function is in fact linear. Thus, $A_{1}=A_{0}=A$, and is not dense in $U\left(\mu_{A} R\right)=$ $U(R)$.

The next two sections present theorems in which the hypotheses on $A$ are weakened.

3. Piecewise- $A$ functions. We prove, again as a corollary of 1.3 , a theorem like 2.2 but applied to a vector space; what is needed is a condition (3.1(b)) more permissive than $2.1(\mathrm{~b})$.

3.1 Definition. A function $g \in R^{X}$ is piecewise- $A$ if there is finite $F \subset A$ such that at each point of $X, g$ agrees with some function in $F$.

$g$ is uniformly locally piecewise- $A$ if there is a $\mu_{A}$-uniform cover $\mathcal{Q}$ such that $g$ agrees with a piecewise- $A$ function on each member of $\mathcal{Q}$.

$A_{2}$ (respectively, $A_{3}$ ) stands for the collection of functions in $R^{X}$ which are

(a) finitely $A$-uniform, and

(b) piecewise- $A$ (respectively, uniformly locally piecewise- $A$ ).

3.2 TheOREM. If $A$ is a vector space, then $A_{3}$ is uniformly dense in $U\left(\mu_{A} X\right)$.

The following prepares for application of 1.3:

3.3 Proposition. Let $A$ be a vector space. Then

(a) $A_{2}$ and $A_{3}$ are vector lattices;

(b) $\mu_{A_{2}}=\mu_{A_{3}}=\mu_{A}$;

(c) a family $\mathscr{F}$ is finitely-A-equiuniform if (and only if) $\mathscr{F}$ is finitely- $A_{2}$ equiuniform;

(d) $\left(A_{2}\right)_{0} \subset A_{3}$.

Proof of 3.3. (a) It is easy to see that $A_{2}$ and $A_{3}$ are vector spaces, because $A$ is. They are lattices because at each point, $a \vee b$ and $a \wedge b$ agree with either $a$ or $b$.

(b) is implied by 3.1(a).

(c) The "only if" part is obvious. Conversely, let $\mathscr{F}$ be $F$-equiuniform with finite $F \subset A_{2}$. For $f \in F$, there is finite $G_{f} \subset A$ such that at each point of $X, f$ agrees with some function in $G_{f}$. With $H=\cup\left\{G_{f} \mid f \in F\right\}, \mathscr{F}$ is $H$-equiuniform, hence finitely- $A$-equiuniform.

(d) Let $\mathcal{F}$ be a countable subset of $A_{2}^{+}$which is finitely- $A_{2}$-equiuniform, with coz $\mathscr{F}$ a $\mu_{A_{2}}$-uniform cover of order one. We show $\bigvee \mathcal{F} \in A_{3}:$ By (c) and 1.1, $\bigvee \mathscr{F}$ is finitely- $A$-uniform. By (b), $\operatorname{coz} \mathscr{F}$ is $\mu_{A}$-uniform. Let $g \in \mathscr{F}$, and 
let $F=\{h \in \mathscr{F} \mid \operatorname{coz} h \cap \operatorname{coz} g \neq \varnothing\}$. By "order-one", $F$ has at most three elements, so $\bigvee F \in A_{2}$ by (a). But on the set $\operatorname{coz} g, \bigvee \mathscr{F}=\bigvee F$. So $\bigvee \mathscr{F} \in A_{3}$.

Proof OF 3.2. Using 3.3(a) and (b), then 1.3, $\left(A_{2}\right)_{0}$ is dense in $U\left(\mu_{A} X\right)^{+}$. Using 3.3(a), (b) and (d), $A_{3}$ is dense in $U\left(\mu_{A} X\right)$.

4. Compositions. We derive some corollaries concerning functions of the form $G \circ\left(a_{1}, \ldots, a_{p}\right)$, where $\left(a_{1}, \ldots, a_{p}\right): X \rightarrow R^{p}$ is the evaluation (or parametric) map defined by $\left(a_{1}, \ldots, a_{p}\right)(x)=\left(a_{1}(x), \ldots, a_{p}(x)\right)$, and $G$ is a function defined at least on the range $\left(a_{1}, \ldots, a_{p}\right)(X)$. The essential observation is this:

4.1. Proposition. Let $A \subset R^{X}$, and $g \in R^{X}$. Then $g$ is finitely- $A$-uniform (2.1) iff $g=G \circ\left(a_{1}, \ldots, a_{p}\right)$ for some $a_{1}, \ldots, a_{p} \in A$ and $G \in$ $U\left(\left(a_{1}, \ldots, a_{p}\right)(X)\right)$.

Proof. If $g=G \circ\left(a_{1}, \ldots, a_{p}\right)$, with $G$ uniformly continuous, then certainly $g \in U\left(\mu_{F} X\right)$ for $F=\left\{a_{1}, \ldots, a_{p}\right\}$.

Let $g \in U\left(\mu_{F} X\right)$, for $F=\left\{a_{1}, \ldots, a_{p}\right\} \subset A$. Clearly, if $a_{i}(x)=a_{i}(y)$ for $i=1, \ldots, p$, then $g(x)=g(y)$. Thus, defining $G:\left(a_{1}, \ldots, a_{p}\right)(X) \rightarrow R$ by $G\left(\left(a_{1}(x), \ldots, a_{p}(x)\right)\right)=g(x)$ makes sense. Let $\varepsilon>0$. Since $g \in U\left(\mu_{F} X\right)$, there is $\delta>0$ with $g^{-1} \delta(\varepsilon)>\wedge a_{i}^{-1} \delta(\delta)$. Now,

$$
\begin{aligned}
G^{-1} \delta(\varepsilon) & =\left(a_{1}, \ldots, a_{p}\right) g^{-1} \delta(\varepsilon)>\left(a_{1}, \ldots, a_{p}\right) \wedge a_{i}^{-1} \delta(\delta) \\
& =\left(\bigwedge \pi_{i}^{-1} \delta(\delta)\right) \cap\left(a_{1}, \ldots, a_{p}\right)(X) .
\end{aligned}
$$

This last is a uniform cover, so is $G^{-1} \delta(\varepsilon)$, and $G$ is uniformly continuous.

Given $A \subset R^{X}$, let comp $A$ denote the class of functions $g$ described in 3.1. Note that, here, we are not assuming $A$ to have any algebraic properties.

4.2 Theorem. For any $A \subset R^{X}, \operatorname{comp}_{1} A$ is uniformly dense in $U\left(\mu_{A} X\right)$.

PRoOF. comp $A$ is itself a vector lattice: for example, $a_{1}+a_{2}=$ $G \circ\left(a_{1}, a_{2}\right)$, where $G(x, y)=x+y$; the other operations go similarly.

It is clear that a finitely comp $A$-uniform function is finitely- $A$-uniform, hence by 4.1 , in $\operatorname{comp} A$. This shows that $(\operatorname{comp} A)_{1}=\operatorname{comp} A$, so by 2.2 , comp $A$ is dense in $U\left(\mu_{\text {comp } A} X\right)$. But, of course, $\mu_{\text {comp } A}=\mu_{A}$.

In a sense, 4.2 reduces the problem of describing $U\left(\mu_{A} X\right)$ to the problem for subsets of $R^{p}(p=1,2, \ldots)$. One feels that one knows more about the functions in $U\left(R^{p}\right)$ than about those in $U(S)$ for $S \subset R^{p}$. So cases in which one can reduce to this may be worth considering.

4.3 Corollary. $\left\{\left\{G \circ\left(a_{1}, \ldots, a_{p}\right) \mid p \in N ; a_{1}, \ldots, a_{p} \in A ; G \in\right.\right.$ $\left.B U\left(R^{p}\right)\right\}$ is dense in $\left.B U\left(\mu_{A} X\right)\right\}$. 
Proof. If $f \in U\left(\mu_{A} X\right)$, then by $4.2, f$ is approximable by a composition $G \circ\left(a_{1}, \ldots, a_{p}\right)$ with $G \in U\left(\left(a_{1}, \ldots, a_{p}\right)(X)\right)$. If $f$ is bounded, $G$ is also bounded and thus extends over all of $R^{p}$ by the Katětov Theorem [K].

4.4 Corollary. $U\left(\mu_{A} X\right)=\left\{G \circ\left(a_{n}\right) \mid\left\{a_{n}\right\} \subset A ; G \in U\left(\left(a_{n}\right)(X)\right)\right\}$ and $B U\left(\mu_{A} X\right)=\left\{G \circ\left(a_{n}\right) \mid\left\{a_{n}\right\} \subset A ; G \in B U\left(R^{\kappa_{0}}\right)\right\}$ (where the sets $\left\{a_{n}\right\}$ are countable).

Proof. The inclusions $\supset$ are automatic.

If $f \in U\left(\mu_{A} X\right)$, then by $4.2, f$ is the uniform limit of a sequence $\left\{f_{n}\right\}$, where $f_{n}=F_{n} \circ\left(a_{1}^{n}, \ldots, a_{p_{n}}^{n}\right)$. It is easily arranged inductively that if $m<n$, then $p_{m}<p_{n}$ and for $i<p_{m}, a_{i}^{m}=a_{i}^{n}$. We thus write $f_{n}=F_{n} \circ\left(a_{1}, \ldots, a_{p_{n}}\right)$. Now let $G_{n} \in U\left(\left(a_{n}\right)(X)\right)$ be defined by $G_{n}\left(\left(a_{n}(x)\right)\right)=$ $F_{n}\left(\left(a_{1}(x), \ldots, a_{p_{n}}(x)\right)\right)$. Since $g_{n} \rightarrow f$ uniformly, $G_{n}$ converges uniformly to some $G \in U\left(\left(a_{n}\right)(X)\right)$. That $f=G \circ\left(a_{n}\right)$ follows.

The proof for $B U\left(\mu_{A} X\right)$ then uses the Katětov Theorem, as in 4.3.

Finally, we derive a theorem of Isbell [I(1)].

To say that $A$ has continuous composition is to say that if $p \in N, a_{1}, \ldots, a_{p}$ $\in A$ and $G \in C\left(R^{p}\right)$, then $G \circ\left(a_{1}, \ldots, a_{p}\right) \in A$. Assuming only this about $A$, it follows easily that $A$ is a vector lattice and ring, as in the proof of 4.2.

4.5 Corollary. If $A$ has continuous composition, then $A$ is dense in $U\left(\mu_{A} X\right)$.

Proof. Given $f \in U\left(\mu_{A} X\right)$, approximate within $\varepsilon$ by $G \circ\left(a_{1}, \ldots, a_{p}\right)$, using 4.2. Extend $G$ to a uniformly continuous function $G_{1}$ on the closure of $\left(a_{1}, \ldots, a_{p}\right)(X)$, and then continuously over $R^{p}$, to $G_{2}$, by the Tietze Extension Theorem. Then $G \circ\left(a_{1}, \ldots, a_{p}\right)=G_{2} \circ\left(a_{1}, \ldots, a_{p}\right) \in A$.

This proof uses 1.3, of course, Isbell's proof is rather simple.

\section{REFERENCES}

[C] A.. Császár, Gleichmässige Approximation und gleichmässige Stetigheit, Acta. Math. Acad. Sci. Hungar. 20 (1969), 253-261. MR 40 \#6492.

[F](1) J. E. Fenstad, On l-groups of uniformly continuous functions. I, Math. Z. 82 (1963), 434-444. MR 28 \#2432.

[F](2) __, On l-groups of uniformly continuous functions. II, Math. Z. 83 (1964), 46-56. MR 30 \# 1493.

[H] A. W. Hager, Vector lattices of uniformby contimuous functions and some categorical methods in uniform spaces, TOPO 72-General Topology and its Applications, Lecture Notes in Math., vol. 378, Springer-Verlag, Berlin and New York, 1972. MR 50 \# 14678.

[I](1) J. R. Isbell, Algebras of uniformly continuous functions, Ann. of Math. (2) 68 (1958), 96-125. MR 21 \#2177.

[I](2), Uniform spaces, Math. Surveys, no. 12, Amer. Math. Soc., Providence, R. I., 1964. MR 30 \#561. 
[K] M. Katětov, On real-valued functions in topological spaces, Fund. Math. 38 (1951), 85-91. MR 14, 304.

[M] W. Maak, Eine Verallgemeinerung des Weierstrasschen Approximationssatzes, Arch. Math. 6 (1955), 188-193. MR 16, 1008.

[NB] G. Nöbeling and H. Bauer, Allgemeine Approximationskriterien mit Anwendungen, Jber. Deutsch. Math. Verein 58 (1955), 54-72. MR 17, 605.

Department of Mathematics, Wesleyan University, Middletown, Connecticut 06457 